\title{
Optimal cutoff of pretreatment neutrophil- to-lymphocyte ratio in head and neck cancer patients: a meta-analysis and validation study
}

\author{
Jae-Keun Cho', Myoung Woo Kim ${ }^{1}$, Ick Soo Choi ${ }^{1}$, Uk Yeol Moon ${ }^{2}$, Min-Ji Kim³ ${ }^{3}$ Insuk Sohn ${ }^{3}$, Seonwoo Kim³ \\ and Han-Sin Jeong ${ }^{2^{*}}$ (D)
}

\begin{abstract}
Background: The prognostic role of neutrophil-to-lymphocyte ratio (NLR) has been proposed in head and neck squamous cell carcinoma (HNSCC). However, it is currently unclear which cutoff values of NLR could consistently and independently differentiate HNSCC patients to better and worse prognosis groups.

Methods: We performed a meta-analysis of prognostic significance of pretreatment NLR values, using data extracted from 24 relevant articles. Main outcomes were overall survival (OS) and disease-free survival (DFS) in HNSCC patients. Pooled hazard ratio (HR) and $95 \%$ confidence intervals $(95 \% \mathrm{Cl})$ were calculated using the random effect model for outcomes. Impacts of NLR cutoff values across the studies were assessed with a meta-regression analysis. Results were validated using an independent data set of patients $(n=540)$.

Results: Pretreatment high NLR values above the cutoff were significantly associated with shorter OS (HR $=1.96$, $95 \% \mathrm{Cl}=1.66-2.31)$ and $\mathrm{DFS}(\mathrm{HR}=1.90,95 \% \mathrm{Cl}=1.41-2.54)$. Of note, NLR cutoffs ranging from 1.9 to 6.0 did not affect HR of OS or DFS in meta-regression analyses. In an independent cohort, any NLR cutoff between 2 and 6 produced significant HR of OS, similarly. Instead of binary cutoffs, three subgroups of $\mathrm{NLR}(<2,2$ to 6 , and $\geq 6)$ showed significant differences of OS in survival analyses.

Conclusions: Meta-analyses confirmed that pretreatment NLR values above the cutoff were associated with shorter survival in HNSCC patients. However, the binary cutoffs of NLR values were variable across studies. Rather, pretreatment NLR values below 2 and above 6 using a three-tier classification $(<2,2$ to 6 , and $\geq 6)$ could consistently imply better and worse prognosis in HNSCC patients, which could be readily translated to clinics.
\end{abstract}

Keywords: Head and neck cancer, Inflammatory marker, Neutrophil, Lymphocyte, Outcomes, Prognosis

\section{Background}

Systemic inflammation has proven to be a major contributing factor in cancer development and progression across a number of tumor types [1-4]. Biologically, tumor associated inflammatory response is recognized as one of cancer hallmarks [5]. High degree of systemic inflammation is associated with worse outcomes in

\footnotetext{
* Correspondence: hansin.jeong@gmail.com

2Department of Otorhinolaryngology-Head and Neck Surgery, Samsung Medical Center, Sungkyunkwan University School of Medicine, Seoul 06351, Republic of Korea

Full list of author information is available at the end of the article
}

cancer patients; meanwhile local inflammation with the infiltration of various immune cells around tumors reflects better outcomes [6-9]. As surrogate markers of systemic inflammation, serum albumin, C-reactive protein, neutrophil- and platelet-to-lymphocyte ratios have been investigated widely [10-13].

Systemic inflammation could have a prognostic value in head and neck squamous cell carcinoma (HNSCC) as in other cancers [4]. Neutrophil-to-lymphocyte ratio (NLR) (dividing the absolute neutrophil count by the absolute lymphocyte count) is one of various biomarkers of systemic inflammation. There have been numerous

(c) The Author(s). 2018 Open Access This article is distributed under the terms of the Creative Commons Attribution 4.0 International License (http://creativecommons.org/licenses/by/4.0/), which permits unrestricted use, distribution, and 
studies on the prognostic role of NLR in various selected populations of HNSCC [14-17]. One clinical advantage using NLR value to estimate prognosis is that it is a simple and readily measurable indicator from routine blood sampling of patients at diagnosis.

However, even with a wealth of reports about the prognostic role of NLR in HNSCC, most studies have adopted cutoff values of NLR driven by internal prognosis grouping without external validation [14-19]. Recent meta-analyses also confirmed the prognostic role of pretreatment NLR value in HNSCC, without suggesting the optimal cutoff value [20-23]. It is currently unclear which cutoff value of NLR could consistently and independently differentiate better and worse prognosis groups in HNSCC patients. Thus, this work was designed to explore whether pre-treatment NLR in HNSCC might have a prognostic significance across multiple studies (meta-analyses), the optimal cutoff values of NLR for consistent differentiation of HNSCC prognosis (evaluation of cutoff point), and whether such cutoff values might be still valid in an independent data set of HNSCC patients (external validation). Through these sequential analyses, we aimed to build a bridge between clinical findings and general application of NLR value in HNSCC.

\section{Methods}

\section{Search strategy}

This meta-analysis was performed according to the recommendations of the Preferred Reporting Items for Systematic Reviews and Meta-Analyses (PRISMA) 2009 guidelines [24]. We systematically searched the MEDLINE, EMBASE and Cochrane library databases focusing on clinical studies published prior to April 30th, 2017. Potentially relevant studies were identified using the following key words: [neutrophil lymphocyte ratio], in combination with [head and neck], [cancer], and [prognosis] (Additional file 1: Table S1). In addition, reference lists of retrieved articles were screened manually to identify additional eligible studies. No language restriction was imposed. Eligibility of these studies was decided through comprehensive reviews and discussions with multiple researchers.

\section{Study selection}

Inclusion criteria for these studies were as follows: (i) enrolled patients had a histological diagnosis of HNSCC, (ii) pre-treatment NLR was calculated from peripheral blood samples before any treatments, and (iii) information about clinical outcomes (disease recurrence, metastatic tumor progression and death from any cause) was available (disease-free survival DFS, progression-free survival PFS, overall survival OS). Studies were excluded if there was insufficient information to calculate the hazard ratio (HR) and $95 \%$ confidence interval $(95 \% \mathrm{CI})$ of outcomes.

\section{Data extraction and quality assessment}

Two authors (JKC, HSJ) independently identified eligible articles and collected the following data: (i) publication information; the first author's name, year of publication, country of study conducted, (ii) pre-treatment NLR data, (iii) clinical features: total patient numbers, TNM stages at diagnosis, disease outcomes and follow-up duration. Any disagreement was resolved by discussion. The Newcastle-Ottawa quality assessment scale for non-randomized studies was implemented to evaluate the quality of included studies (Additional file 1: Table S2) [25].

\section{Meta-analysis for prognostic impacts of pretreatment NLR status}

NLR status, a binary variable (high NLR and low NLR), was defined according to the study-specific NLR cutoff value. To determine the effect of NLR status on clinical outcomes, HRs were pooled using the random effect model [26, 27]. HR of more than 1 indicated worse outcome for the group having NLR above cutoffs compared to the group having NLR below cutoffs. The precision of estimates was quantified by $95 \%$ CI.

Heterogeneity was measured by Higgins and Green $\mathrm{I}^{2}$ test [27]. Values of $\mathrm{I}^{2}$ ranged between 0\% (no heterogeneity) and 100\% (maximal heterogeneity). Heterogeneity of the study was considered to be substantial at $P<0.1$ and $\mathrm{I}^{2}>50 \%$. We also evaluated potential publication bias with Egger's regression test and funnel plot [26]. A sensitivity analysis with a trim-and-fill method was conducted. All above analyses were executed using R 3.3.2 (Vienna, Austria; http://www.r-project.org/) with a package of metafor. A two-sided $P$ value of less than 0.05 was considered statistically significant.

\section{Meta-regression of cutoff values of NLR}

Study-specific NLR cutoff values ranged from 1.9 to 6.0 (median $=3)$. Meta-regression analyses were then performed to determine whether the impact of NLR status on clinical outcomes was different according to each study-specific factor: the cutoff value of NLR, age, gender, tumor stage (I/II versus III/IV), tumor subsite (oral cavity, pharynx, larynx, others) and number of index tumors (single versus multiple). Results are presented as change of HR of OS and DFS.

\section{Validation study using an independent data set}

To confirm the prognostic impact of NLR cutoff values estimated from previous analyses, we conducted a validation study using an independent cohort in our institution. All HNSCC patients were enrolled prospectively into our head and neck cancer registry and they provided written informed consents for use of their clinical and biological data under an Institutional Review Board approved protocol. From registered HNSCC patients, we 
included patients who had been treated for their HNSCC (newly diagnosed) between 2010 and 2014 ( $n=$ 540).(Additional file 2: Raw data for a validation study) The clinical characteristics of our cohort were comparable to the study patients enrolled in the meta-analyses (Additional file 1: Table S3). All patients followed the current standard treatment protocols (The National Comprehensive Cancer Network guideline, http:// www.nccn.org). Patients with secondary cancers, other pathologies, or palliative treatments were excluded. HR on outcomes (OS and DFS) of NLR status according to possible NLR cutoffs in the range of 2 to 6 by increment of 0.1 were calculated in multivariate Cox proportional hazard regression analyses. The present study focused on pretreatment NLR values and clinical outcomes was approved by our Institutional Review Board again before data collection.

\section{Results}

\section{Characteristics of identified studies}

We identified 38 potentially relevant articles through multiple database searches. Among them, 14 were further excluded, mainly due to inability to estimate HR and $95 \% \mathrm{CI}$ of outcomes. The article by Charles et al. [14] presented results with oropharyngeal cancer and non-oropharyngeal cancer separately. We regarded their study results as two independent studies. Thus, 25 observational studies in 24 articles were included in our meta-analysis (Fig. 1). Characteristics of included studies are listed in Table 1 . Among them, 24 reported HRs for
OS outcomes, including 12 and 5 studies for HRs of DFS and PFS, respectively.

\section{Prognostic significance of pretreatment NLR values}

Twenty four eligible studies were analyzed in OS meta-analysis. The total HR of the random effect model was 1.96 [95\%CI: 1.66-2.31] $(P<0.001)$ (Fig. 2). However, substantial heterogeneities across these studies were noted $\left(\mathrm{I}^{2}=48.29 \%, P=0.0053\right)$. Regarding DFS meta-analysis, 12 studies were enrolled. An overall HR was 1.90 [95\%CI: $1.41-2.54](P<0.0001)$, indicating that high NLR value above cutoff was a significant predictor for DFS in HNSCC patients. The heterogeneity of these studies was also significant $\left(\mathrm{I}^{2}=70.4 \%, P=0.0002\right)$.

In terms of outcomes for PFS, there were only five studies describing PFS in their reports. An overall HR for PFS was 1.82 [95\%CI: 1.43-2.33] $(P<0.0001)$, with low heterogeneity $\left(\mathrm{I}^{2}=0.0 \%, P=0.92\right)$ (Additional file 3: Figure $\mathrm{S} 1$ ). Thus, high NLR value (above cutoffs) could predict disease progression in metastatic settings of HNSCC, as well as OS and DFS. Because the number of studies was small $(n=5)$ with an outcome of PFS, we mainly focused on OS and DFS in subsequent analyses.

\section{Evaluation of publication bias and sensitivity analysis}

Next, we evaluated publication bias by Egger's regression test for funnel plot asymmetry. In OS meta-analysis for enrolled studies, the funnel plot showed a relatively asymmetric distribution with Egger's $P$ value of 0.0007 . Similarly, there was a significant publication bias in DFS meta-analysis $(P=0.0017)$ (Fig. 2). However, a trim-and-fill

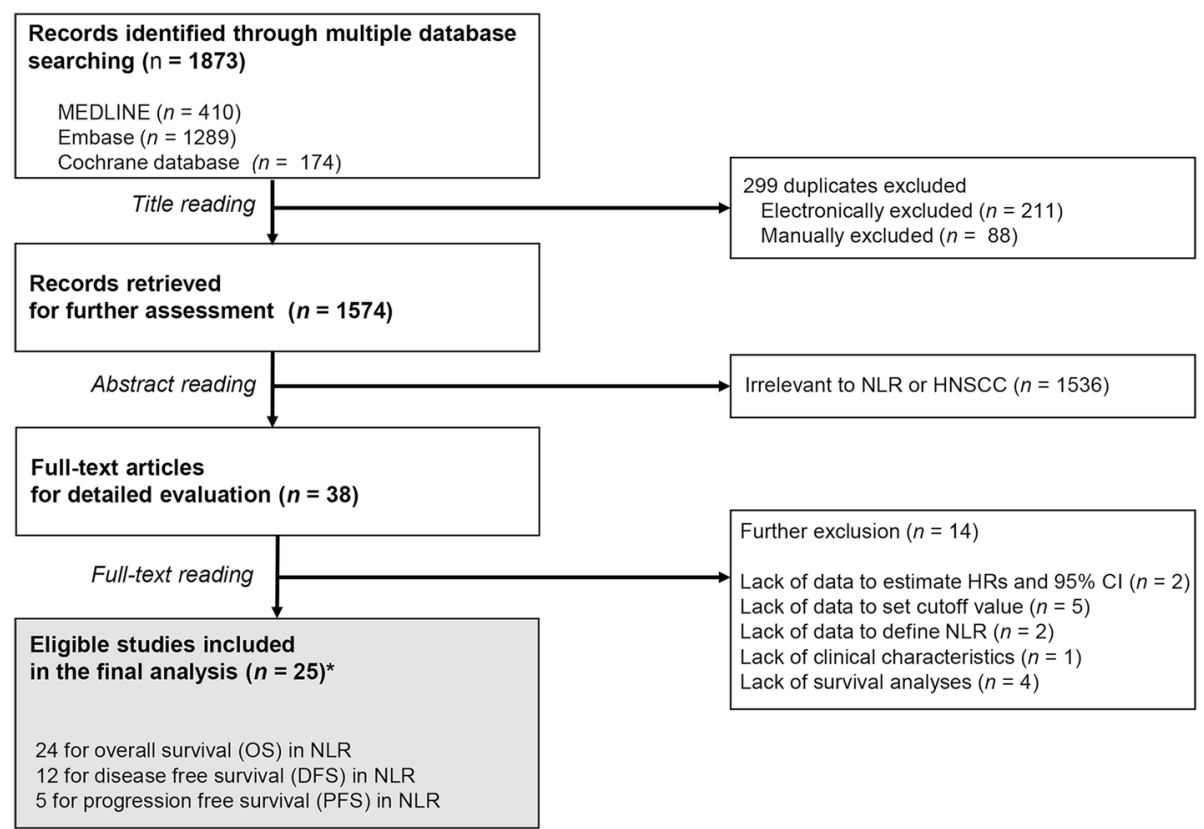

Fig. 1 Flow chart of study selection process ( ${ }^{*} 25$ studies in 24 articles) 
Table 1 Characteristics of studies included in the final analyses

\begin{tabular}{|c|c|c|c|c|c|c|c|c|c|c|}
\hline & Study (First Author) & Publication year & $\begin{array}{l}\text { No. of } \\
\text { subject }\end{array}$ & $\begin{array}{l}\text { NLR } \\
\text { cutoff }\end{array}$ & Stage & Index of tumor & Follow-up (mo) & Outcome & HR extraction & $\begin{array}{l}\text { Multivariate } \\
\text { adjustment }\end{array}$ \\
\hline 1 & $\mathrm{He}[28]$ & 2012 & 1410 & 2.17 & I-IV & Pharyn $x^{a}$ & 41 & OS, PFS & Reported & Yes \\
\hline 2 & Millrud [29] & 2012 & 20 & 6 & I-IV & Others $^{b}$ & 24 & OS & Reported & No \\
\hline 3 & Fang [30] & 2013 & 226 & 2.44 & I-IV & Oral cavity & & OS, DFS & Reported & No \\
\hline 4 & Rassouli [18] & 2013 & 273 & 4.27 & & Others & 45 & DFS & Reported & No \\
\hline 5 & $\operatorname{Jin}[31]$ & 2014 & 229 & 3.6 & III, IV & Pharynx & & OS & Reported & Yes \\
\hline 6 & Young [32] & 2014 & 249 & 5 & & Pharynx & 46 & OS & Reported & No \\
\hline 7 & Haddad [33] & 2015 & 46 & 5 & III, IV & Others & 34 & OS, DFS & Reported & No \\
\hline 8 & Rachidi [34] & 2015 & 543 & 4.39 & I-IV & Others & 64 & OS & Reported & Yes \\
\hline 9 & Salim [35] & 2015 & 79 & 2.93 & I-IV & Others & & OS, PFS & Reported & No \\
\hline 10 & Selzer [36] & 2015 & 170 & 5 & I-IV & Others & & OS & Reported & Yes \\
\hline 11 & Song [37] & 2015 & 146 & 2.3 & & Pharynx & 26 & OS & Reported & No \\
\hline 12 & Sun [19] & 2015 & 251 & 2.7 & I-IV & Pharynx & 50 & OS, PFS & Reported & Yes \\
\hline 13 & Tu [16] & 2015 & 141 & 2.17 & I-IV & Larynx & 51 & OS, DFS & Reported & Yes \\
\hline 14 & Charles [14] $(1)^{c}$ & 2016 & 76 & 5 & I-IV & Pharynx & 29 & OS, DFS & Reported & Yes \\
\hline 15 & Charles [14] (2) & 2016 & 69 & 5 & I-IV & Others & 29 & OS, DFS & Reported & Yes \\
\hline 16 & Chua [38] & 2016 & 380 & 3 & I-IV & Pharynx & & OS, DFS & Reported & Yes \\
\hline 17 & Fu [39] & 2016 & 420 & 2.59 & III, IV & Larynx & & OS & Reported & Yes \\
\hline 18 & Ikeguchi [40] & 2016 & 59 & 5 & III, IV & Pharynx & 38 & OS & Reported & Yes \\
\hline 19 & Kano [41] & 2016 & 285 & 1.92 & I-IV & Others & 63 & OS, DFS & Reported & Yes \\
\hline 20 & Kim [42] & 2016 & 104 & 3 & III, IV & Others & 39 & OS, DFS & Reported & Yes \\
\hline 21 & Moon [43] & 2016 & 153 & 3 & I-IV & Others & 39 & OS, PFS & Reported & Yes \\
\hline 22 & Nakashima [15] & 2016 & 124 & 2.4 & IIII, IV & Pharynx & 47 & OS, DFS & Reported & Yes \\
\hline 23 & Wong [17] & 2016 & 140 & 3.1 & I-IV & Larynx & 41 & OS, DFS & Reported & Yes \\
\hline 24 & Zeng [44] & 2016 & 115 & 3 & III, IV & Larynx & 45 & OS, PFS & Reported & Yes \\
\hline 25 & Turri_Zanoni [45] & 2017 & 215 & 5.56 & & Others & 51 & OS, DFS & Reported & Yes \\
\hline
\end{tabular}

$N L R$ neutrophil to lymphocyte ratio, $P L R$ platelet to lymphocyte ratio, OS overall survival, DFS disease free survival, $P F S$ progression free survival ainclude following tumor subsite; nasopharynx, oropharynx and hypopharynx

${ }^{b}$ include following tumor subsite; nasal cavity or not specified

'A paper by Charles et al. had clinical data with two groups; oropharyngeal cancer and non-oropharyngeal cancer. We divided the results into two independent sets and employed these results separately into our analyses, named as Charles (1) and Charles (2)

method, in which we calculated the overall HR by making additional data set symmetrical to y-axis according to the midpoint of funnel plot, did not reverse results of the random effect model, confirming that there was no significant difference in the outcome (Table 2, Additional file 4: Figure S2).

\section{Meta-regression of cutoff values of NLR}

Through the meta-analyses described above, we confirmed the prognostic significance of pretreatment NLR status for OS and DFS in HNSCC. However, cutoff values of NLR for dividing HNSCC patients into high and low NLR groups, ranged from 1.9 to 6.0 in enrolled studies. Thus, we next determined whether the effect of NLR status on clinical outcomes was different according to the cutoff value of NLR. To address this, we applied meta-regression for the association between the HRs of OS (or DFS) and study-specific NLR cutoff values (Fig. 3).

NLR cutoff values of 1.9 to 6.0 did not affect the extracted HRs of OS or DFS ( $P=0.16$ for OS; $P=0.18$ for DFS). In short, absolute NLR cutoff values between 1.9 and 6.0 did not seem to matter, although groups below and above NLR cutoffs did show significant survival differences. Among other potential factors, only age variable had influenced the HRs of NLR status in OS and DFS (Additional file 1: Table S4). A prognostic significance of NLR status did not differ according to tumor subsites in the head and neck, in addition to gender, tumor stage, and multiplicity.

\section{A validation study using an independent data set}

Next, we conducted an external validation study. For this, we used 540 registered HNSCC patients in our 


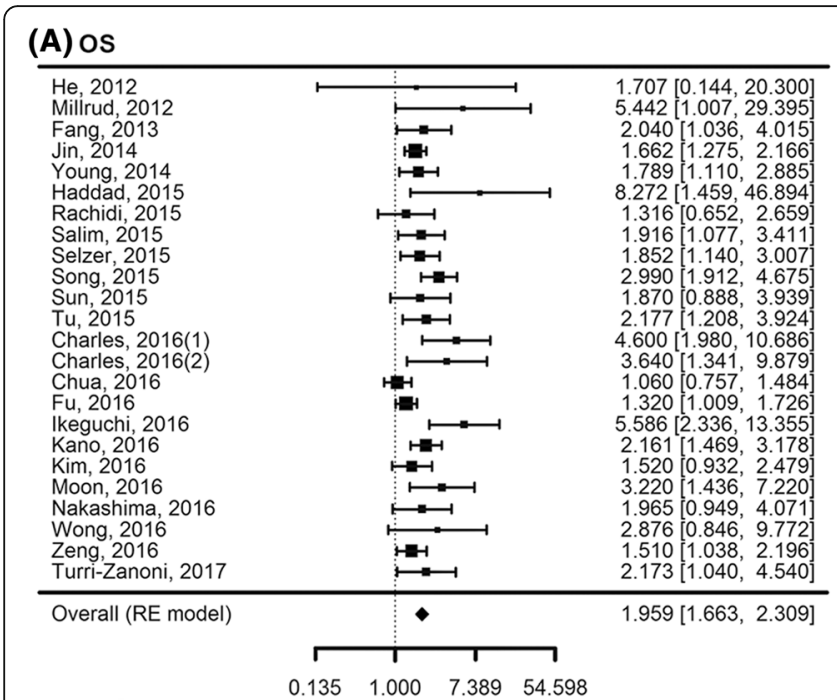

(C) os

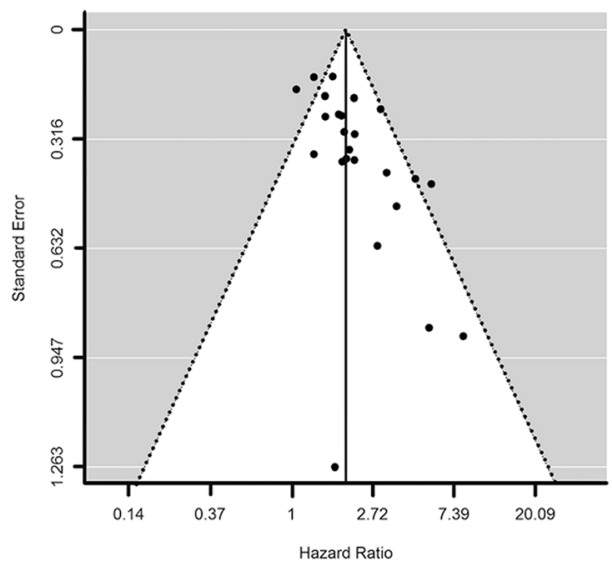

(B) DFS

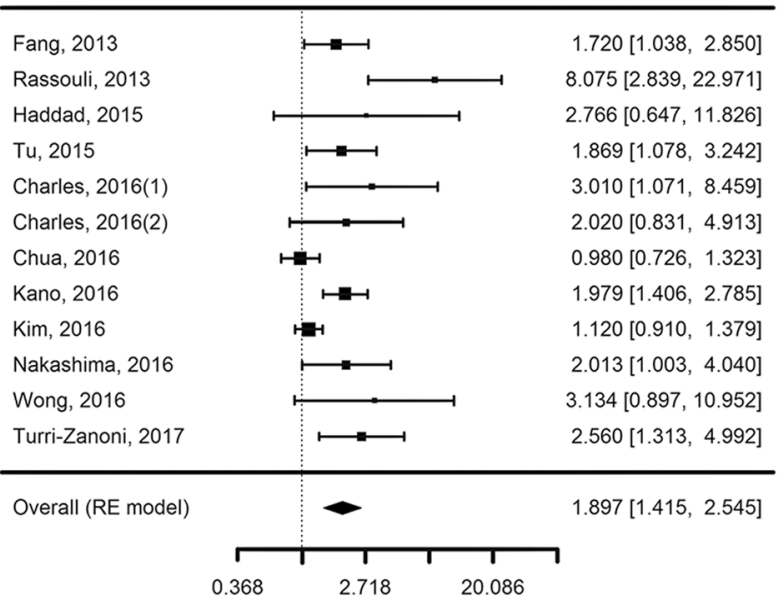

(D) DFS

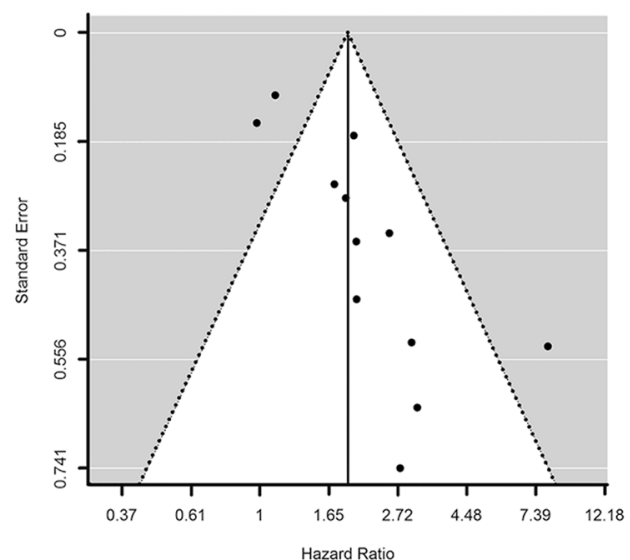

Fig. 2 Forest plots illustrating prognostic significance of NLR value on overall survival a and disease-free survival $\mathbf{b}$ in HNSCC patients (The first author name of published article, Publication year). Numbers indicated the hazard ratios (HR) of survival outcomes with [95\% confidence interval]. $H R>1$ indicated worse outcome for the group having NLR above cutoffs compared to the group having NLR below cutoffs. OS: Overall survival, DFS: Disease-free survival, RE model: Random effect model. c Funnel plots of analyzed studies according to overall and disease-free survivals (d)

institute. In the independent cohort, we evaluated the prognostic impact of NLR status according to NLR cutoffs in the range between 2.0 and 6.0 by increment of 0.1 . The HRs of OS were significant in any NLR cutoff values between 2.0 and 6.0 (to 7.8$)(P<0.001)$ after adjusting for age, gender, TNM stage and tumor site (a multivariate Cox hazard regression model), in line with results of the previous meta-regression analyses (Fig. 3). However, the HRs of DFS were not significant in NLR cutoffs from 1.5 to 8.5 in our cohort $(P=0.089)$.

Table 2 Results of sensitivity analysis

\begin{tabular}{|c|c|c|c|c|c|c|c|}
\hline \multicolumn{3}{|l|}{ NLR values } & \multicolumn{2}{|l|}{ Random effects model } & \multicolumn{2}{|c|}{ Heterogeneity } & \multirow{2}{*}{$\begin{array}{l}\text { Egger's regression test } \\
\text { for funnel plot asymmetry } \\
P \text {-value }\end{array}$} \\
\hline Outcomes & Method & No. of articles & Hazard ratio (95\% Cl) & $P$-value & $P$-value & I squared (\%) & \\
\hline \multirow[t]{2}{*}{ OS } & Raw & 24 & $1.96(1.66-2.31)$ & $<0.0001$ & 0.0053 & $48.29 \%$ & 0.0007 \\
\hline & Trim-and-fill & 33 & $1.63(1.35-1.97)$ & $<0.0001$ & $<0.0001$ & $65.38 \%$ & 0.5874 \\
\hline \multirow[t]{2}{*}{ DFS } & Raw & 12 & $1.90(1.41-2.54)$ & $<0.0001$ & 0.0002 & $70.39 \%$ & 0.0017 \\
\hline & Trim-and-fill & 17 & $1.53(1.12-2.08)$ & 0.0068 & $<0.0001$ & $75.53 \%$ & 0.2780 \\
\hline
\end{tabular}



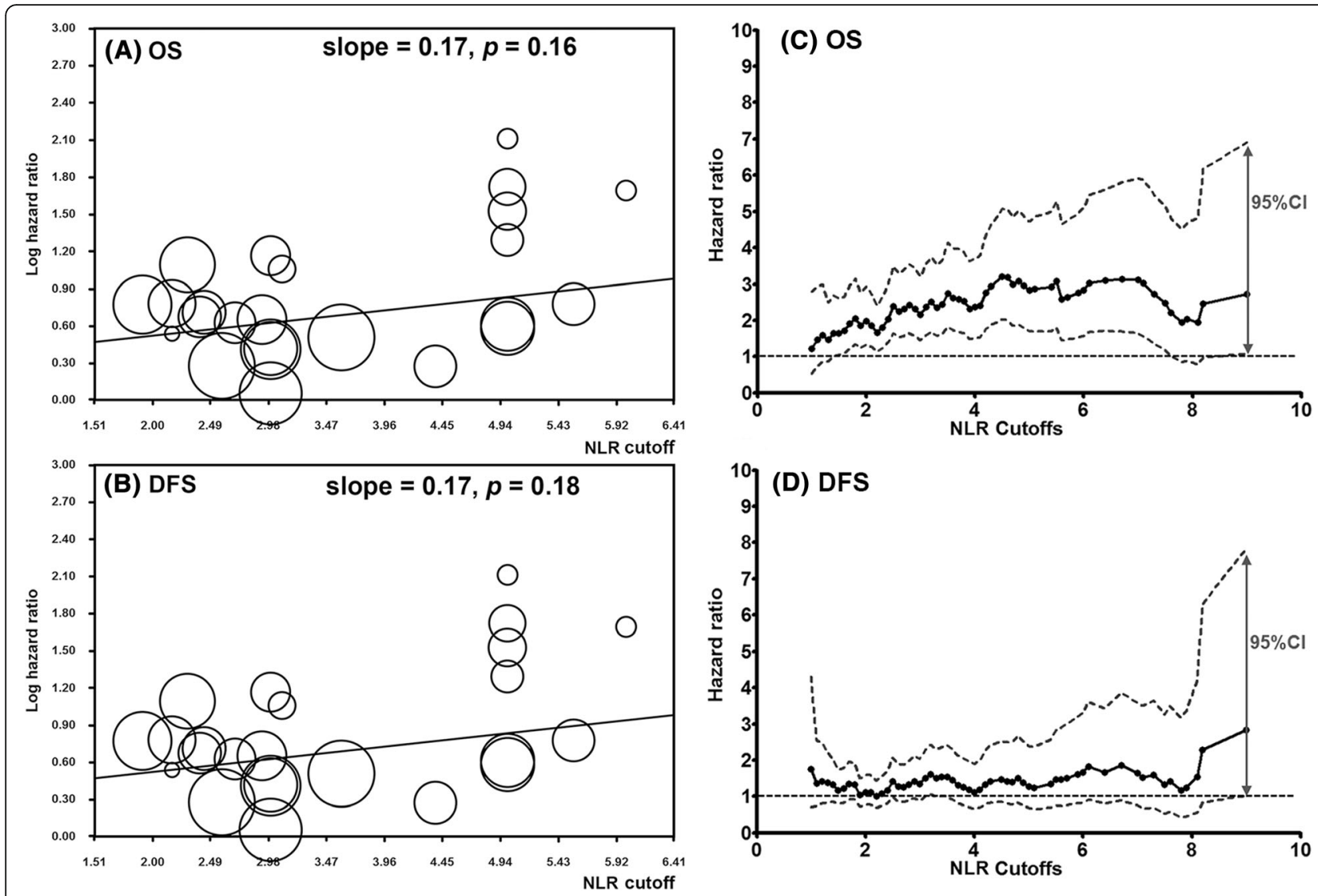

Fig. 3 Meta-regression plots of NLR hazard ratio according to NLR cutoffs. a OS, b DFS. The center and radius of each circle indicated the Log(HR) and 95\% Cl of enrolled studies. X-axis meant the study-specific NLR cutoff values. c-d Hazard ratio (HR) and 95\% Cl according to NLR cutoffs by multivariate Cox proportional hazard regression model in an independent HNSCC cohort. c OS, d DFS. Solid line: HR, Dashed line: $95 \% \mathrm{Cl}$. HR $>1$ and $95 \% \mathrm{Cl}>1$ indicated significant worse outcome for the group having NLR above cutoffs compared to the group having NLR below cutoffS

In other words, any NLR cutoff between 2.0 and 6.0 produced significant discrimination for better and worse prognosis group in terms of OS. For a more practical application of NLR status on prognosis estimation in HNSCC patients, our data suggested a three-tier classification of NLR status (NLR: <2.0, 2.0-6.0, $\geq 6.0$ ), instead of binary NLR grouping based on a single NLR cutoff. Our results also confirmed a significant discrimination of OS among groups of below NLR 2.0 (reference), NLR 2.0 to $6.0(\mathrm{HR}=1.80$, [95\% CI: $1.13-2.86])$ and above NLR $\geq 6.0(\mathrm{HR}=3.92$, [95\% CI: 1.89-8.12]) in our cohort by a multivariate Cox proportional hazard model (adjusting for age, gender, TNM stage and subsite) (Fig. 4).

\section{Discussion}

Our meta-analyses confirmed the prognostic impact of pretreatment NLR value in HNSCC patients. NLR is a readily measurable indicator that can be obtained from patient blood at diagnosis. Thus, it could be easily incorporated into prognosis grouping in HNSCC patients. However, its universal application is hindered by different
NLR cutoffs across studies. Most studies have adopted their own cutoff values of NLR driven by internal prognosis grouping without external validation [14-19]. Thus, we conducted this study to investigate universal optimal cutoff value of NLR for consistent differentiation of HNSCC prognosis.

NLR cutoff values in the published articles have been variable among published articles, ranging from 1.9 to 6.0. Interestingly, our meta-regression analysis revealed that NLR status according to any NLR cutoffs between 1.9 and 6.0 had similar prognostic impact on OS and DFS. In a validation cohort, we observed consistent results in terms of OS outcome. Thus, it is hard to determine a single specific cutoff value between 2.0 and 6.0 as a NLR cutoff for binary OS prognosis grouping. Rather, it indirectly suggests a bimodal distribution of patients (i.e., large proportions of patients with better prognosis below NLR $=2.0$ and large portion of patients with worse prognosis above NLR $=6.0$ ). As a practical conclusion with prognostic impact of pretreatment NLR on HNSCC patients, our results showed that three-tier categorization (NLR values $<2.0,2.0$ to $6.0, \geq 6.0$ ) would 
(A) OS

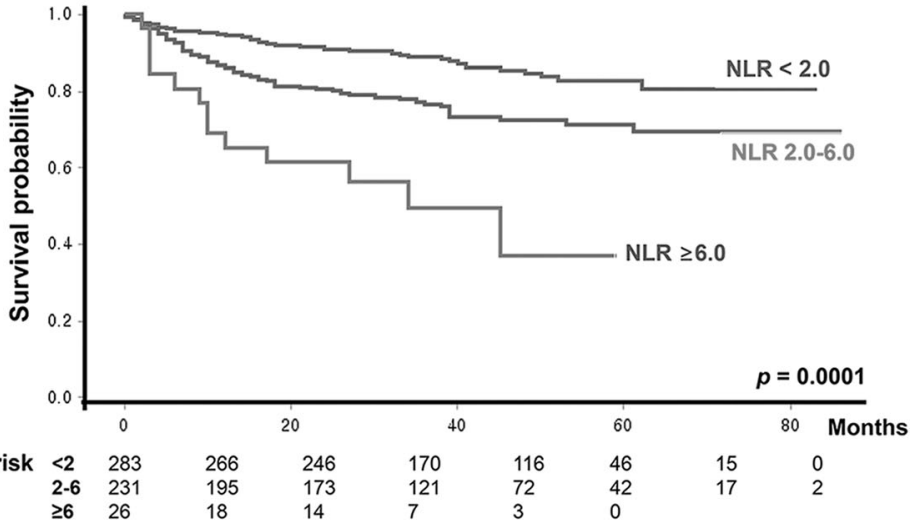

(B) DFS

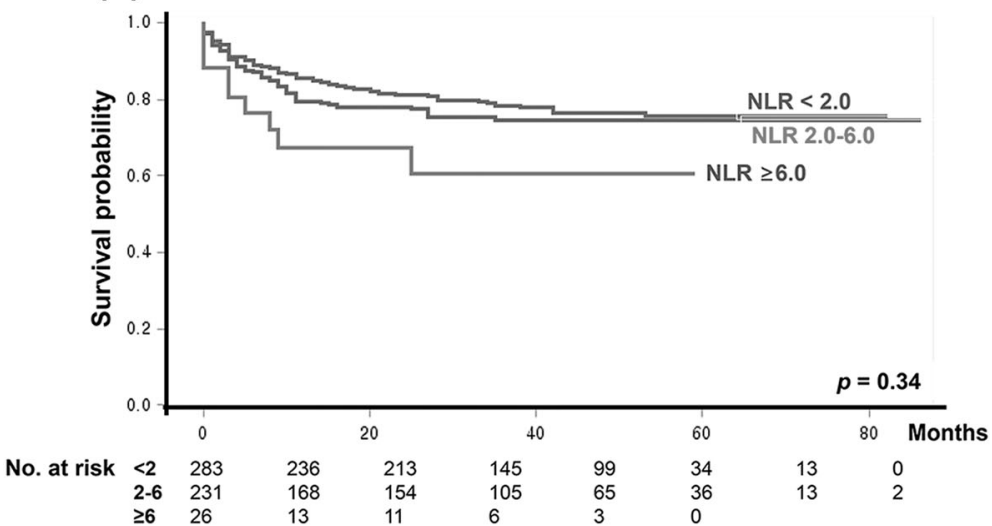

Fig. 4 Survival difference among groups of below NLR 2.0, NLR 2 to 6 and $\geq$ NLR 6.0 in a validation cohort. a OS, b DFS

be more clinically relevant and easy to be translated it to clinics.

One thing to note was that our results from this meta-analysis were not validated in our cohort in terms of DFS outcomes. Reasons for this discrepancy include different distribution of HNSCC subsites, treatment-related factors and patient factors. Systemic inflammation may reflect host response to cancer associated inflammation or immune reaction $[1,2,4]$. Thus, it is reasonable to think that NLR status might be more related to patient overall outcomes, than to local tumor control. For example, dysfunctional larynx due to laryngeal cancer or treatments can cause patient mortality (aspiration pneumonia) without metastatic disease. In such case, we frequently observe elevated inflammatory markers (for example, C-reactive protein). However, this assumption needs to be investigated further in the future studies.

Although we employed a statistical method of meta-analysis and performed validation with an independent cohort, our study still had some limitations to draw a solid conclusion. First, there were the heterogeneity issue and publication bias of articles included in our meta-analyses. A significant heterogeneity across these studies was found in both DFS and OS meta-analyses except for PFS analysis. Similarly, there was a significant publication bias in DFS and OS meta-analysis. Thus, these enrolled studies seemed not to be representative of NLR significance in HNSCC, and the positive results of NLR values on prognostic impacts might be published more in the literature than the negative results of NLR values. To minimize bias, we adopted a random effect model to estimate the overall HR of DFS and OS and performed a sensitivity analysis (trim-and-fill method) to confirm our primary analyses in this study. Nevertheless, the further studies with less heterogeneity and publication bias are needed to confirm our conclusion.

Head and neck cancers include a group of cancers arising from the various sites in the head and neck. In this study, we employed multivariate analyses adjusting tumor subsites in the meta-regression analysis (Additional file 1: Table S4) and in the validation set (Fig. 4), and confirmed that a prognostic significance of NLR status did not differ according to tumor subsites in the head and neck. 
However, the validation cohort did not include the patients with nasopharyngeal cancer (Additional file 1: Table S3). Thus, our results should be re-evaluated with a site-specific prospective cohort of head and neck cancers. In addition, enrolled studies in meta-analyses did not report the human papilloma virus (HPV) or p16 status in HNSCC. Thus, we could not analyze the significance of NLR values according to the HPV status of HNSCC in this study.

\section{Conclusions}

Pretreatment high NLR values above the cutoff were significantly associated with shorter survival in HNSCC patients. NLR values below two and above six could consistently differentiate better and worse prognosis in HNSCC patients, which might be readily translated to clinics.

\section{Additional files}

Additional file 1: Table S1. Searching strategy. Table S2. Quality assessment of included studies (Newcastle-Ottawa scale). Table S3. Clinical characteristics of a validation cohort $(n=540)$. Table S4. Meta-regression models for hazard ratios (HR) of NLR status. (DOCX $26 \mathrm{~kb}$ )

Additional file 2: Raw data for validation study. (XLSX 71 kb)

Additional file 3: Figure S1. Prognostic significance of NLR value on progression-free survival (PFS) in HNSCC patients (The first author name of published article, Publication year). (A) Forest plot, (B) Funnel plot. Numbers indicated the hazard ratios (HR) of survival outcomes with [95\% confidence interval]. HR > 1 indicated worse outcome for the group having NLR above cutoffs compared to the group having NLR below cutoffs. RE model: Random effect model. (TIF 2385 kb)

Additional file 4: Figure S2. A sensitivity analysis to adjust publication bias. (A) OS, (B) DFS. (TIF 3062 kb)

\section{Funding}

This work was supported by a grant of the National Research Foundation of Korea (NRF) funded by the Korea government (MEST) (No. 2015R1D1A1A09056771), a 2015 Inje University research grant (No. 20151089) and a 2016 basic-clinical collaborative research grant (No. SMX1161461) funded by Samsung Biomedical Research Institute (SBRI). The above funders had no role in study design, in data collection, data analysis, data interpretation, writing of the manuscript, or decision to submit this manuscript for publication.

\section{Availability of data and materials}

All data generated or analyzed during this study are included in the Additional files.

\section{Authors' contributions}

Conception and design: JKC, HSJ. Data collection: JKC, MWK, ISC, UYM. Analysis and interpretation: JKC, MWK, ISC, UYM, MJK, IS, SK, HSJ. Statistical analysis: MJK, IS, SK. Critical revision of the article: JKC, MJK, IS, SK, HSJ. Study supervision: HSJ. All authors have read and approved the manuscript.

\section{Authors' information}

JK Cho, MW Kim, IS Choi: Department of Otorhinolaryngology-Head and Neck Surgery, Inje University Ilsan Paik Hospital, Inje University School of Medicine, Goyang, Republic of Korea, UY Moon, HS Jeong: Department of Otorhinolaryngology-Head and Neck Surgery, Samsung Medical Center, Sungkyunkwan University School of Medicine, Seoul, Republic of Korea. MJ Kim, I Sohn, S Kim: Statistics and Data Center, Research Institute for Future Medicine, Samsung Medical Center, Seoul, Republic of Korea.

\section{Ethics approval and consent to participate}

The study protocol was approved by the Institutional Review Board of Samsung Medical Center. All patients submitted the written informed consents for use of their clinical and biological data at enrollment of our head and neck cancer registry. The data used in this study was de-identified.

\section{Consent for publication}

Not applicable.

\section{Competing interests}

The authors declare that they have no competing interests.

\section{Publisher's Note}

Springer Nature remains neutral with regard to jurisdictional claims in published maps and institutional affiliations.

\section{Author details}

${ }^{1}$ Department of Otorhinolaryngology-Head and Neck Surgery, Inje University Ilsan Paik Hospital, Inje University School of Medicine, Goyang, Republic of Korea. ${ }^{2}$ Department of Otorhinolaryngology-Head and Neck Surgery, Samsung Medical Center, Sungkyunkwan University School of Medicine, Seoul 06351, Republic of Korea. ${ }^{3}$ Statistics and Data Center, Research Institute for Future Medicine, Samsung Medical Center, Seoul, Republic of Korea.

Received: 19 June 2018 Accepted: 30 September 2018

Published online: 11 October 2018

\section{References}

1. Aggarwal BB, Vijayalekshmi RV, Sung B. Targeting inflammatory pathways for prevention and therapy of cancer: short-term friend, long-term foe. Clin Cancer Res. 2009;15(2):425-30.

2. Grivennikov SI, Greten FR, Karin M. Immunity, inflammation, and cancer. Cell. 2010;140(6):883-99.

3. O'Callaghan DS, O'Donnell D, O'Connell F, O'Byrne KJ. The role of inflammation in the pathogenesis of non-small cell lung cancer. J Thorac Oncol. 2010;5(12):2024-36.

4. Templeton AJ, McNamara MG, Seruga B, Vera-Badillo FE, Aneja P, Ocana A, Leibowitz-Amit R, Sonpavde G, Knox JJ, Tran B, et al. Prognostic role of neutrophil-to-lymphocyte ratio in solid tumors: a systematic review and meta-analysis. J Natl Cancer Inst. 2014;106(6):dju124.

5. Hanahan D, Weinberg RA. Hallmarks of cancer: the next generation. Cell. 2011;144(5):646-74.

6. Acharyya S, Oskarsson T, Vanharanta S, Malladi S, Kim J, Morris PG, ManovaTodorova K, Leversha M, Hogg N, Seshan VE, et al. A CXCL1 paracrine network links cancer chemoresistance and metastasis. Cell. 2012;150(1):165-78.

7. Eruslanov EB, Bhojnagarwala PS, Quatromoni JG, Stephen TL, Ranganathan A, Deshpande C, Akimova T, Vachani A, Litzky L, Hancock WW, et al. Tumorassociated neutrophils stimulate $T$ cell responses in early-stage human lung cancer. J Clin Invest. 2014;124(12):5466-80

8. Fridlender ZG, Sun J, Kim S, Kapoor V, Cheng G, Ling L, Worthen GS, Albelda SM. Polarization of tumor-associated neutrophil phenotype by TGF-beta: "N1" versus "N2" TAN. Cancer Cell. 2009;16(3):183-94.

9. Mantovani A, Allavena P, Sica A, Balkwill F. Cancer-related inflammation. Nature. 2008;454(7203):436-44

10. Guthrie GJ, Charles KA, Roxburgh CS, Horgan PG, McMillan DC, Clarke SJ. The systemic inflammation-based neutrophil-lymphocyte ratio: experience in patients with cancer. Crit Rev Oncol Hematol. 2013;88(1):218-30.

11. McMillan DC. The systemic inflammation-based Glasgow prognostic score: a decade of experience in patients with cancer. Cancer Treat Rev. 2013;39(5):534-40.

12. Roxburgh CS, McMillan DC. Role of systemic inflammatory response in predicting survival in patients with primary operable cancer. Future Oncol. 2010;6(1):149-63.

13. Vigano A, Bruera E, Jhangri GS, Newman SC, Fields AL, Suarez-Almazor ME. Clinical survival predictors in patients with advanced cancer. Arch Intern Med. 2000;160(6):861-8.

14. Charles KA, Harris BD, Haddad CR, Clarke SJ, Guminski A, Stevens M, Dodds T, Gill AJ, Back M, Veivers D, et al. Systemic inflammation is an independent predictive marker of clinical outcomes in mucosal squamous cell carcinoma of the head and neck in oropharyngeal and non-oropharyngeal patients. BMC Cancer. 2016;16:124. 
15. Nakashima H, Matsuoka Y, Yoshida R, Nagata M, Hirosue A, Kawahara K, Sakata J, Arita H, Hiraki A, Nakayama H. Pre-treatment neutrophil to lymphocyte ratio predicts the chemoradiotherapy outcome and survival in patients with oral squamous cell carcinoma: a retrospective study. BMC Cancer. 2016;16:41.

16. Tu XP, Qiu QH, Chen LS, Luo XN, Lu ZM, Zhang SY, Chen SH. Preoperative neutrophil-to-lymphocyte ratio is an independent prognostic marker in patients with laryngeal squamous cell carcinoma. BMC Cancer. 2015;15:743.

17. Wong BY, Stafford ND, Green VL, Greenman J. Prognostic value of the neutrophil-to-lymphocyte ratio in patients with laryngeal squamous cell carcinoma. Head Neck. 2016;38(Suppl 1):E1903-8.

18. Rassouli A, Saliba J, Castano R, Hier M, Zeitouni AG. Systemic inflammatory markers as independent prognosticators of head and neck squamous cell carcinoma. Head Neck. 2015;37(1):103-10.

19. Sun W, Zhang L, Luo M, Hu G, Mei Q, Liu D, Long G, Hu G. Pretreatment hematologic markers as prognostic factors in patients with nasopharyngeal carcinoma: neutrophil-lymphocyte ratio and platelet-lymphocyte ratio. Head Neck. 2016;38(Suppl 1):E1332-40.

20. Takenaka Y, Oya R, Kitamiura T, Ashida N, Shimizu K, Takemura K, Yamamoto Y, Uno A. Prognostic role of neutrophil-to-lymphocyte ratio in head and neck cancer: a meta-analysis. Head Neck. 2018;40(3):647-55.

21. Tham T, Bardash Y, Herman SW, Costantino PD. Neutrophil-to-lymphocyte ratio as a prognostic indicator in head and neck cancer: a systematic review and meta-analysis. Head Neck. 2018.

22. Yu Y, Wang H, Yan A, Wang H, Li X, Liu J, Li W. Pretreatment neutrophil to lymphocyte ratio in determining the prognosis of head and neck cancer: a meta-analysis. BMC Cancer. 2018;18(1):383.

23. Yin J, Qin Y, Luo YK, Feng M, Lang JY. Prognostic value of neutrophil-tolymphocyte ratio for nasopharyngeal carcinoma: a meta-analysis. Medicine (Baltimore). 2017:96(29):e7577.

24. Moher D, Liberati A, Tetzlaff J, Altman DG, Group P. Preferred reporting items for systematic reviews and meta-analyses: the PRISMA statement. Int J Surg. 2010;8(5):336-41.

25. The Newcastle-Ottawa Scale (NOS) for assessing the quality of nonrandomised studies in meta-analyses. http://www.ohri.ca/programs/ clinical_epidemiology/oxford.asp. Accessed 30 Apr 2017.

26. Egger M, Davey Smith $G$, Schneider M, Minder C. Bias in meta-analysis detected by a simple, graphical test. Bmj. 1997;315(7109):629-34.

27. JPT H, Green S. Cochrane handbook for systematic reviews of interventions version 5.1.0 [updated March 2011]: The Cochrane Collaboration; 2011.

28. He JR, Shen GP, Ren ZF, Qin H, Cui C, Zhang Y, Zeng YX, Jia WH. Pretreatment levels of peripheral neutrophils and lymphocytes as independent prognostic factors in patients with nasopharyngeal carcinoma. Head Neck. 2012;34(12):1769-76.

29. Millrud CR, Mansson Kvarnhammar A, Uddman R, Bjornsson S, Riesbeck K, Cardell LO. The activation pattern of blood leukocytes in head and neck squamous cell carcinoma is correlated to survival. PLoS One. 2012;7(12):e51120.

30. Fang HY, Huang XY, Chien HT, Chang JT, Liao CT, Huang JJ, Wei FC, Wang $\mathrm{HM}$, Chen $\mathrm{IH}$, Kang $\mathrm{CJ}$, et al. Refining the role of preoperative C-reactive protein by neutrophil/lymphocyte ratio in oral cavity squamous cell carcinoma. Laryngoscope. 2013;123(11):2690-9.

31. Jin $Y, Y e X$, He C, Zhang B, Zhang Y. Pretreatment neutrophil-to-lymphocyte ratio as predictor of survival for patients with metastatic nasopharyngeal carcinoma. Head Neck. 2015;37(1):69-75.

32. Young CA, Murray $\sqcup$, Karakaya E, Thygesen HH, Sen M, Prestwich RJ. The prognostic role of the neutrophil-to-lymphocyte ratio in oropharyngeal carcinoma treated with Chemoradiotherapy. Clin Med Insights Oncol. 2014;8:81-6.

33. Haddad CR, Guo L, Clarke S, Guminski A, Back M, Eade T. Neutrophil-tolymphocyte ratio in head and neck cancer. J Med Imaging Radiat Oncol. 2015;59(4):514-9.

34. Rachidi S, Wallace K, Wrangle JM, Day TA, Alberg AJ, Li Z. Neutrophil-tolymphocyte ratio and overall survival in all sites of head and neck squamous cell carcinoma. Head Neck. 2016;38(Suppl 1):E1068-74.

35. Salim DK, Mutlu H, Eryilmaz MK, Salim O, Musri FY, Tural D, Gunduz S, Coskun HS. Neutrophil to lymphocyte ratio is an independent prognostic factor in patients with recurrent or metastatic head and neck squamous cell cancer. Mol Clin Oncol. 2015;3(4):839-42.

36. Selzer E, Grah A, Heiduschka G, Kornek G, Thurnher D. Primary radiotherapy or postoperative radiotherapy in patients with head and neck cancer: comparative analysis of inflammation-based prognostic scoring systems. Strahlenther Onkol. 2015;191(6):486-94.
37. Song Y, Liu H, Gao L, Liu X, Ma L, Lu M, Gao Z. Preoperative neutrophil-tolymphocyte ratio as prognostic predictor for hypopharyngeal squamous cell carcinoma after radical resections. J Craniofac Surg. 2015;26(2):e137-40.

38. Chua ML, Tan SH, Kusumawidjaja G, Shwe MT, Cheah SL, Fong KW, Soong YL, Wee JT, Tan TW. Neutrophil-to-lymphocyte ratio as a prognostic marker in locally advanced nasopharyngeal carcinoma: a pooled analysis of two randomised controlled trials. Eur J Cancer. 2016;67:119-29.

39. Fu Y, Liu W, OuYang D, Yang A, Zhang Q. Preoperative neutrophil-tolymphocyte ratio predicts long-term survival in patients undergoing Total laryngectomy with advanced laryngeal squamous cell carcinoma: a singlecenter retrospective study. Medicine (Baltimore). 2016;95(6):e2689.

40. Ikeguchi M. Glasgow prognostic score and neutrophil-lymphocyte ratio are good prognostic indicators after radical neck dissection for advanced squamous cell carcinoma in the hypopharynx. Langenbeck Arch Surg. 2016; 401(6):861-6.

41. Kano S, Homma A, Hatakeyama H, Mizumachi T, Sakashita T, Kakizaki T, Fukuda S. Pretreatment lymphocyte-to-monocyte ratio as an independent prognostic factor for head and neck cancer. Head Neck. 2017;39(2):247-53.

42. Kim DY, Kim IS, Park SG, Kim H, Choi YJ, Seol YM. Prognostic value of posttreatment neutrophil-lymphocyte ratio in head and neck squamous cell carcinoma treated by chemoradiotherapy. Auris Nasus Larynx. 2017:44(2):199-204.

43. Moon H, Roh JL, Lee SW, Kim SB, Choi SH, Nam SY, Kim SY. Prognostic value of nutritional and hematologic markers in head and neck squamous cell carcinoma treated by chemoradiotherapy. Radiother Oncol. 2016:118(2):330-4.

44. Zeng YC, Chi F, Xing R, Xue M, Wu LN, Tang MY, Wu R. Pre-treatment neutrophil-to-lymphocyte ratio predicts prognosis in patients with locoregionally advanced laryngeal carcinoma treated with chemoradiotherapy. Jpn J Clin Oncol. 2016;46(2):126-31.

45. Turri-Zanoni M, Salzano G, Lambertoni A, Giovannardi M, Karligkiotis A, Battaglia P, Castelnuovo P. Prognostic value of pretreatment peripheral blood markers in paranasal sinus cancer: neutrophil-to-lymphocyte and platelet-to-lymphocyte ratio. Head Neck. 2017;39(4):730-6.

Ready to submit your research? Choose BMC and benefit from

- fast, convenient online submission

- thorough peer review by experienced researchers in your field

- rapid publication on acceptance

- support for research data, including large and complex data types

- gold Open Access which fosters wider collaboration and increased citations

- maximum visibility for your research: over $100 \mathrm{M}$ website views per year

At $\mathrm{BMC}$, research is always in progress.

Learn more biomedcentral.com/submissions 